\title{
A 12-Week Multi-Domain Lifestyle Modification to Reduce Depressive Symptoms in Older Adults: A Preliminary Report
}

\author{
Ki Jung Chang', Chang Hyung Hong'2, Hyun Woong Roh², Kang Soo Lee', \\ Eun Hee Lee ${ }^{2}$, Jinju Kim ${ }^{4}$, Hyun Kook Lim ${ }^{5} \bowtie$, and Sang Joon Son ${ }^{2} \bowtie$ \\ ${ }^{1}$ Department of Psychiatry, Ajou Good Hospital, Suwon, Republic of Korea \\ ${ }^{2}$ Department of Psychiatry, Ajou University School of Medicine, Suwon, Republic of Korea \\ ${ }^{3}$ Department of Psychiatry, CHA University School of Medicine, CHA Hospital, Seongnam, Republic of Korea \\ ${ }^{4}$ Department of Psychiatry, Ajou Danam Hospital, Suwon, Republic of Korea \\ ${ }^{5}$ Department of Psychiatry, Yeouido St. Mary's Hospital, College of Medicine, The Catholic University of Korea, Seoul, Republic of Korea
}

Objective The objective of this study was to compare the effectiveness of usual care management (UCM) and a newly-developed lifestyle modification with contingency management (LMCM) for geriatric depressive symptoms in the community.

Methods A randomized controlled trial was conducted in 93 older adults with major depressive disorder at community mental health centers. A 12 week multi-domain LMCM was developed by providing positive reinforcement using 'gold medal stickers' as a symbolic incentive to motivate their participation and adherence. Participants were allocated to LMCM ( $n=47)$ and UCM ( $n=46)$ groups. They were then subjected to the 12 week treatment. Effects of the two intervention methods on Geriatric Depression Scale were determined using mixed model analysis.

Results Participants in the LMCM group had greater decline in GDS score per month than participants in the UCM group after adjusting for age, sex, years of education, living alone, and MMSE scores at baseline examination [coefficient for GDS score (95\% CI): -1.08 (-1.51, -0.65), $\mathrm{p}<0.001$, reference: UCM group].

Conclusion LMCM is safe and easy to use with a low cost. LMCM is suitable as psychosocial intervention for older adults with depressive symptoms in the community.

Psychiatry Investig 2018;15(3):279-284

Key Words Community, Psychosocial intervention, Depressive symptoms, Elderly.

\section{INTRODUCTION}

Depression is one of the major causes of disability for the elderly. It accounts for $1.6 \%$ of total disability. ${ }^{1}$ There are three representative approaches to treat depression: medication, psychological intervention, and lifestyle modification. ${ }^{2-4}$ While medication and psychological intervention are first line treat-

Received: July 14, 2017 Accepted: August 10, 2017

$\triangle$ Correspondence: Sang Joon Son, MD, PhD

Department of Psychiatry, Ajou University School of Medicine, 164 World cupro, Yeongtong-gu, Suwon 16499, Republic of Korea

Tel: +82-31-219-5180, Fax: +82-31-219-5179, E-mail: sjsonpsy@ajou.ac.kr

$\triangle$ Correspondence: Hyun Kook Lim, MD, PhD

Department of Psychiatry, Yeouido St. Mary's Hospital, College of Medicine, The Catholic University of Korea, 10 63-ro, Yeongdeungpo-gu, Seoul 07345, Republic of Korea

Tel: +82-2-3779-1250, Fax: +82-2-780-6577, E-mail: drblues@catholic.ac.kr

(c) This is an Open Access article distributed under the terms of the Creative Commons Attribution Non-Commercial License (http://creativecommons.org/licenses/by$\mathrm{nc} / 4.0$ ) which permits unrestricted non-commercial use, distribution, and reproduction in any medium, provided the original work is properly cited. ments for depression, lifestyle modification offers a potentially safe and low-cost option. It is especially suitable for elderly populations in the community. ${ }^{4}$

Lifestyle modification for managing depression involves the application of environmental, behavioral, and psychological concepts to increase physical and mental wellness. ${ }^{5}$ This may involve modification of several domain related to depression, including diet, physical activity, relaxation, recreation, and avoidance of smoking and alcohol. ${ }^{4,6,7}$ Especially, scheduling meaningful activities which encourage community engagement and strengthen social relationship is a psychosocial technique that can improve depression. ${ }^{8,9}$

However, lifestyle modification in geriatric patients should be considered in the context of easy applicability and effectiveness for the elderly in the community. Motivational issues, time and resources restrictions, and priority issue might have impacts on older adult's ability to implement lifestyle changes in the community. ${ }^{10}$ First, it is necessary to consider 
readiness to habitual behavior change regarding lifestyle modification. ${ }^{4}$ Second, continuous and active engagement is needed when developing and planning of intervention. ${ }^{11}$ Third, intervention should be individually tailored. It should offer incentives to improve motivation because lack of motivation is frequently found in older patients with depression. ${ }^{12}$

Previously, we have proposed a therapeutic model to change the lifestyle of the elderly by reducing cognitive dysfunction though taking cognizance of factors mentioned above. ${ }^{8}$ Based on the previous model, we developed a community-implantable multi-domain lifestyle modification model with contingency management to reduce depression in older adults. The main objective of the present study was to conduct a randomized controlled trial to test the effectiveness, feasibility, and acceptability of this intervention compared to usual care management for older adults with depression in the community.

\section{METHODS}

\section{Participants}

The study was conducted from August 2015 to January 2016 for adults with depression in geriatric community mental health centers located at Suwon and Gwangju, Republic of Korea. The study protocol registered at ClinicalTrial.gov (NCT03095820). These community centers had outreach sites throughout the city's districts. They provide a range of social, health, educational, and recreational services for the elderly. A total of 93 participants with mean age of $77.8 \pm 6.6$ years ( $77.1 \%$ females) were recruited through these community agencies. Inclusion criteria for participants were: 1) those with non-psychotic, unipolar MDD DSM-IV diagnosis (Mini-International Neuropsychiatric Interview) ${ }^{13}$; 2) those with Montgomery Asberg Depression Rating Scale (MADRS) score of 17 or higher ${ }^{14}$; and 3 ) those who were taking antidepressants at stable dosage for at least 6 weeks prior to study entry without any medical recommendation for medication change for the next 3 months. Pharmacotherapy was uncontrolled and provided by community physicians. Exclusion criteria were: 1 ) those with other Axis I psychiatric disorder; 2) those with acute or severe medical illness (e.g., metastatic cancer, liver failure); 3) those who were taking drugs known to cause depression; 4) those with advanced dementia; and 5) those with aphasia or inability to speak Korean. Subjects used in this study were solely of Asian ethnicity (Korean). The study was approved by the Institutional Review Board of the Ajou University (AJIRB-SBR-SUR5-132). All participants provided signed informed consent.

\section{Randomization and masking}

Randomization was designed in blocks of four participants using SAS. The study coordinator sequentially allocated par- ticipants to either usual care management (UCM) or lifestyle modification with contingency management (LMCM). The allocation ratio was 1:1. Raters were independent evaluators who were unaware of randomization status or study hypotheses. Participants were unaware of study hypotheses either.

\section{Therapist training and treatment fidelity}

The intervention was carried out by trained health worker in mental health community center. For treatment fidelity, they received training on brief advising process, assessing activity level using a simple self-assessment tool, providing how to increase the activity level, and selecting adequate lifestyle modification goals. A manual was also provided to health workers with systematic introductions. Health workers followed the study's written protocols when making any intervention-related recommendations.

\section{Intervention}

We developed a 12 week multi-domain LMCM. Prize-based contingency management is an approach based on operant conditioning theory. ${ }^{15}$ It encourages and maintains desirable behavior change by providing positive reinforcement for recovery of depressive symptoms. ${ }^{15}$ Prizes for positive reinforcement in this study were symbolic gold medal stickers to motivate participation and increase sustainability of desirable behavior for depression. ${ }^{8}$ Although the most commonly used incentives have been financial, we have developed a symbolic incentive that is simple. It can be easily and widely provided in the community.

Based on SIGN guidelines for non-pharmaceutical management of depression, the following four lifestyle factors related to geriatric depressive symptoms were managed: physical activity, healthy diet, social activity, and emotional regulation. ${ }^{4,16}$ Lifestyle modification message included information on deleterious effects of geriatric depression and the importance of engaging in moderate physical activity, maintaining healthy diet, social activity, and emotional regulation to prevent it. Through detailed initial interviews with participants, simple individual goals of prize-based contingency management for each element were set. For example, physical activity goals involved encouraging participants to perform at least $30 \mathrm{~min}$ of moderate physical activity 3 times per week. The most frequently recommended type of activity was walking. Healthydiet recommendation consisted of encouraging participants to consume high quantity of fruit and vegetables, control their weight, have intake of fish or meat at least twice a week, and so on. To encourage social activity, participants were provided with information about the value of an active life style from the perspective of social connectedness and taking part in available recreational community services for the elderly. Emo- 
tional regulation management consisted of relaxation and recreation to improve mood and balance work-rest-play dynamic. Relaxation and recreational activities provided opportunity to direct their mind away from rumination and worry. To maintain the motivation of participants, prize-based contingency management was applied for each lifestyle modification goal. Once a week of telephone-check and once a month of visitcheck were provided to each participant during the follow-up period of 12 weeks. Evaluations were conducted at baseline and at each visit.

UCM was used as a control condition. ${ }^{17,18}$ UCM was a telephone (once a week) and home-delivered (once a month) supportive psychotherapy administered in 12 weekly sessions focusing on non-specific therapeutic factors such as facilitating expression of effect, conveying empathy, and imparting optimism. To parallel the delivery of LMCM, community health worker provided UCM to participants in a conventional manner.

\section{Measurements}

Two clinicians (investigators) agreed on the diagnosis after reviewing SCID-R and other ratings. Certified raters performed in-home assessments at study entry (baseline) and at 4, 8, and 12 weeks of the study period. Depressive symptoms in the elderly were assessed using the Korean version of the Geriatric Depression Scale (GDS)-Short Form. ${ }^{19}$ It was a self-report assessment validated for use in elderly Korean subjects. The questionnaire consisted of 15 Yes/No questions related to depression. A cut-off point of 8 exhibiting a sensitivity of $85 \%$ and a specificity of $69 \%$ for diagnosing a major depressive episode as compared to the Diagnostic and Statistical Manual of Mental Disorders, Fourth Edition-Text Revision (DSM-IV-TR) ${ }^{19}$ was used in this study. This evaluation for self-rated depression using GDS was confirmed by trained health worker in mental health community center.

Questionnaires were conducted at baseline to evaluate the following: 1) demographic characteristics (including age, sex, and years of education); 2) medical illness; and 3) cognitive burden (including scores of the Korean version of the MiniMental State Examination-MMSE ${ }^{20}$ ).

\section{Sample size determination}

Based on a between treatment effect size of 0.70 for depression and disability, we predicted that with at least 36 participants per group and $13 \%$ attrition rate, we would have at least 0.80 power at 0.05 two-tailed significance level with ICC $=0.40$.

\section{Statistical analyses}

Descriptive statistics were used to estimate frequencies, means, and standard deviations of collected variables. Inde- pendent group's t-test was used to analyze continuous variables while chi-square test was used to analyze categorical variables. Mixed-effects linear regression analyses (LMM) and repeated measure of ANOVA were used to compare GDS scores (baseline and at 4, 8, and 12 weeks) between UCM and LMCM groups. The model included a random intercept fixed effect for intervention, time, and interventions by time interactions. Age, gender, years of education, living alone, and cognitive function were included as covariates in the model. Missing GDS values were imputed with fitted values derived from regression models following the intent to treat principle. $\mathrm{p}$-values of less than 0.05 were considered statistically significant. All statistical analysis were performed using Predictive Analytics Software (PASW) version 22.0 (SPSS Inc., Chicago, IL, USA).

\section{RESULTS}

\section{Participants characteristics}

A total of 125 individuals were screened and 93 were found to be eligible. They were randomized to UCM ( $n=46)$ vs. LMCM ( $n=47)$ groups for 3 monthly sessions (Figure 1). Of these 93 participants, $12(12.9 \%)$ were males and $81(87.1 \%)$ were females. The mean age of these participants was $77.8 \pm 6.6$ years. The mean number for years of education was $4.4 \pm 3.9$ years. Their average MMSE score was $23.2 \pm 3.7$, which was higher than the cutoff for potential dementia (cut-off value of 17 to 18). Baseline demographic and clinical characteristics of these 93 participants are summarized in Table 1. In baseline examination, there was no significant difference in age, sex, years of education, living alone, cognitive function, or baseline GDS score between subjects who were receiving UCM and those who were receiving LMCM (Table 1). All participants in UCM and LMCM groups were depressed according to cut off point of GDS score (at 8). Of these 93 participants randomized, 88 (94.6\%) completed the evaluation (UCM, 44 of $46,95.7 \%$; LMCM, 44 of 47, 93.5\%). There was no significant difference in demographics, cognitive function, and depressive symptoms between subjects who dropped out and those who completed assessments.

\section{Change in depressive symptoms between two interventions}

As shown in Figure 2, the UCM group showed a 1.93-point decline in GDS score while the LMCM group had a 4.98-point decline in GDS score during the 12 weeks of intervention. Improvement in depressive symptoms from baseline to the end of 3-month session was higher in the LMCM group compared to that in the UCM group (repeated measure of ANOVA: $\mathrm{F}=19.43$, $\mathrm{p}<0.001$ between UCM and LMCM groups). Moreover, differences in GDS scores between UCM and LMCM 


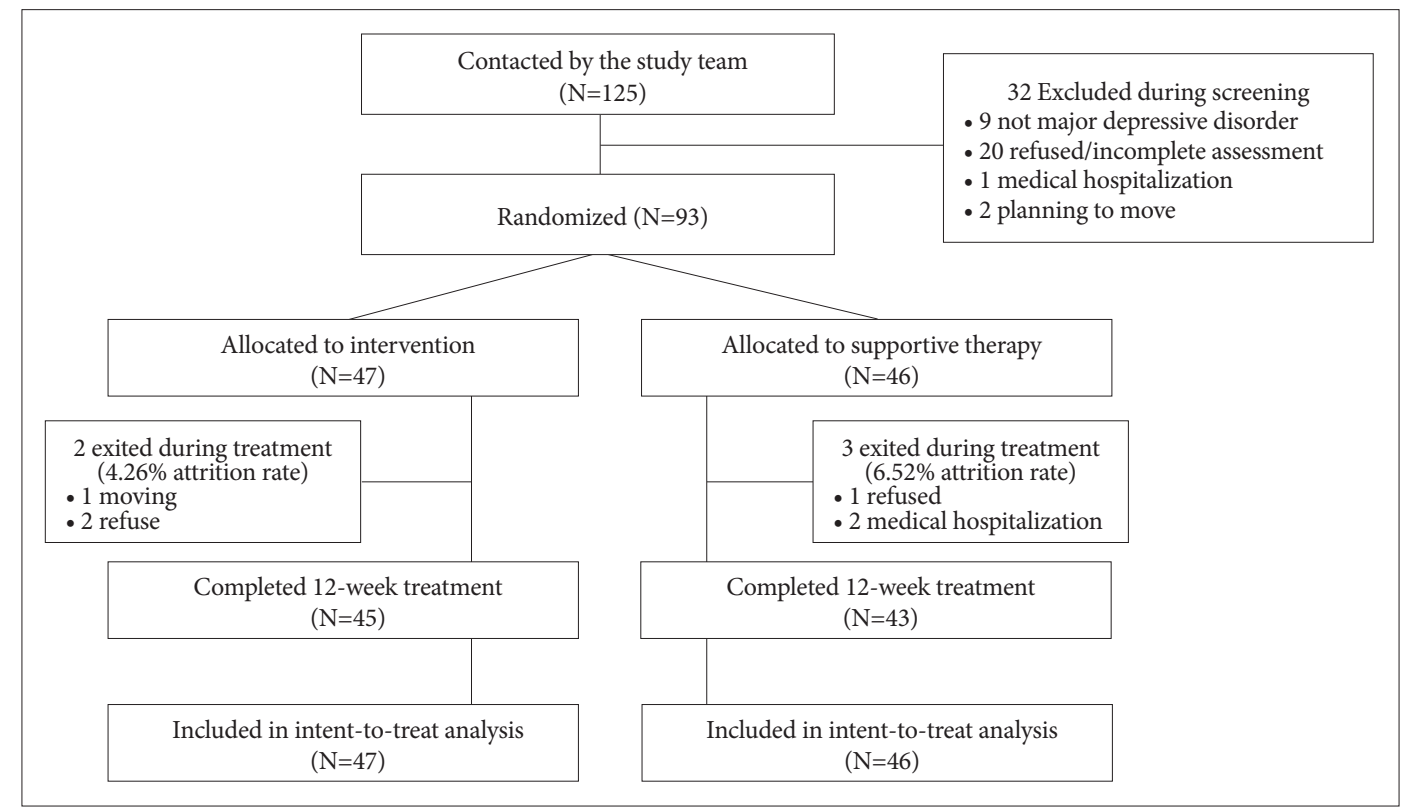

Figure 1. Flow diagram for lifestyle modification with contingency management.

Table 1. Demographics of participants according to UCM and LMCM at baseline

\begin{tabular}{|c|c|c|c|c|c|}
\hline Variable & Total $(\mathrm{N}=93)$ & $\mathrm{UCM}(\mathrm{N}=46)$ & $\operatorname{LMCM}(\mathrm{N}=47)$ & $\chi^{2}$ or $t$ & $\mathrm{p}$ \\
\hline Age, years & $77.8 \pm 6.6$ & $77.0 \pm 7.2$ & $78.5 \pm 6.0$ & -1.036 & 0.303 \\
\hline Sex (male), N (\%) & $12(12.9)$ & $8(17.4)$ & $4(8.5)$ & 1.631 & 0.231 \\
\hline Years of education & $4.4 \pm 3.9$ & $4.5 \pm 4.1$ & $4.2 \pm 3.8$ & 0.404 & 0.687 \\
\hline Living alone, $\mathrm{N}(\%)$ & $50(53.8)$ & $20(43.5)$ & $30(63.8)$ & 4.505 & 0.105 \\
\hline MMSE & $23.2 \pm 3.7$ & $23.0 \pm 3.9$ & $23.4 \pm 3.6$ & -0.546 & 0.586 \\
\hline Baseline GDS & $12.3 \pm 2.2$ & $12.2 \pm 2.2$ & $12.5 \pm 2.2$ & -0.596 & 0.553 \\
\hline
\end{tabular}

UCM: Usual Care Management, LMCM: Lifestyle Modification with Contingency Management

group were prominent only at 12 weeks among the four assessment time points of the study (UCM: 10.2 \pm 3.6 , LMCM: $7.5 \pm 4.1, \mathrm{t}=3.45, \mathrm{p}=0.001$ at 12 weeks), although depressive symptoms reduction was observed in both intervention groups by the end of the study. When GDS scores of subjects were categorized into two groups (normal, GDS score of 0-7; depressed, GDS score of 8 or higher), remission rate of geriatric depression was $19.6 \%$ (9/46) in the UCM group and $46.8 \%$ (22/47) in the LMCM group. Difference in the remission rate between the two intervention groups was statistically significant $\left(\chi^{2}=7.76, \mathrm{df}=1, \mathrm{p}=0.005\right)$.

In mixed-effects model consisting of intervention group, time, and intervention group by time interaction as fixed effects with intercept and slope as random effects, significant differences in the decline of GDS score were also observed by the end of the study. Participants in the LMCM group had greater decline (by approximately 1.08 in GDS scores per month) than participants in the UCM group after adjusting for age, sex, years of education, living alone, and MMSE scores at baseline examination [coefficient for GDS score (95\% CI):
-1.08 (-1.51, -0.65), $\mathrm{p}<0.001$, reference: UCM group] (Table 2). In addition to total GDS score, effects of the two intervention methods on subscale of GDS (pessimism, lowered affect, and lack of motivation) were also examined. In mixed model analysis, pessimism, lowered effect, and lack of motivation subscales of GDS had greater improvement in the LMCM group compared to those in the UCM group [coefficient for subscale of GDS (95\% CI), pessimism: $-0.61(-0.85,-0.37), \mathrm{p}<0.001$; lowered affect: $-0.24(-0.43,-0.06), \mathrm{p}=0.01$; lack of motivation: $-0.22(-0.37,-0.06), \mathrm{p}=0.01]$.

\section{DISCUSSION}

The main finding of this study was that health worker-initiated psychosocial intervention with symbolic rewards was superior to primary health worker offered-usual care management for geriatric depressive symptoms in the community. By the end of the study, the remission rate in the LMCM group was approximately 2.4 times higher than that in the UCM group. These results were unchanged after adjusting for de- 


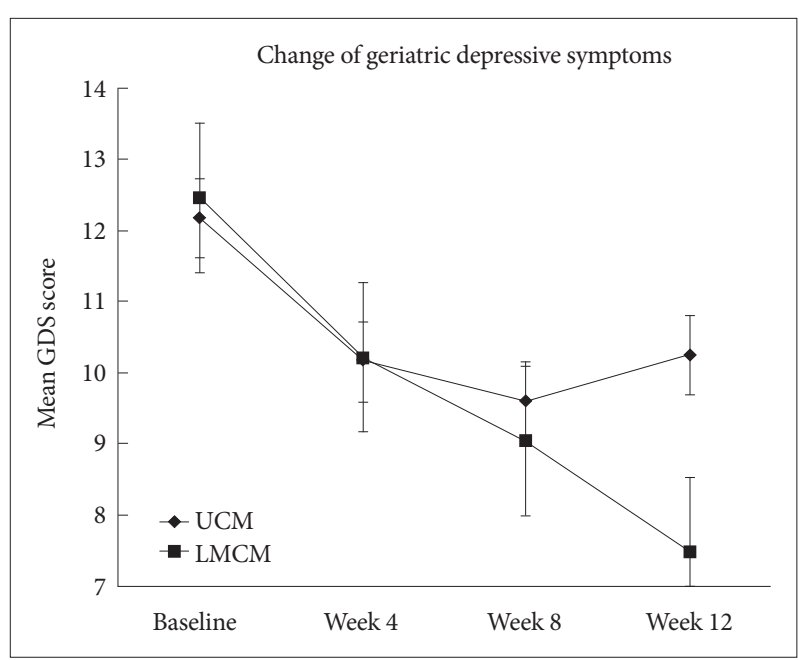

Figure 2. Change of geriatric depressive symptoms according to interventions. UCM: Usual Care Management, LMCM: Lifestyle Modification with Contingency Management.

Table 2. Regression coefficients in the mixed effect models for total and subtotal of GDS score influenced by UCM and LMCM

\begin{tabular}{lll}
\hline \multicolumn{1}{c}{ GDS } & Coefficient & p-value \\
\hline GDS total & & \\
UCM $\times$ time & Reference & $<0.001$ \\
LMCM $\times$ time & -1.082 & \\
Pessimism & & \\
UCM $\times$ time & Reference & $<0.001$ \\
LMCM $\times$ time & -0.608 & \\
Lowered affect & & \\
UCM $\times$ time & Reference & 0.010 \\
LMCM $\times$ time & -0.243 & \\
Lack of motivation & & \\
$\mathrm{UCM} \times$ time & Reference & 0.006 \\
LMCM $\times$ time & -0.219 & \\
\hline
\end{tabular}

Adjusted for age, sex, years of education, living alone and MMSE. UCM: Usual Care Management, LMCM: Lifestyle Modification with Contingency Management

mographic factors and quantitative measure of general cognitive function. Moreover, the tendency of gradual decrease in depressive symptoms over time in the LMCM group was maintained at 12 weeks, while such decrease was not maintained in the UCM group at 12 weeks.

The effect of community-based usual care management on elderly patients with depression has been reported in previous studies. ${ }^{21,22}$ Most community based care management for older adults consisted of providing health care-related information, connecting to mental health care service system, and providing psychoeducation and supportive psychotherapy. ${ }^{23}$ However, our approach had an emphasis on symbolic incentives as well as mental health to promote lifestyle modification- related psychotherapy, thus improving geriatric depressive symptoms. Simple counseling by only advising elderly patients about their mental health is difficult to change their behaviors. It might increase resistance to change their depressive symptoms due to their lack of motivation. ${ }^{9,24,25}$ Supportive and continuous encouragement from primary health workers with contingency management using symbolic incentives might increase their engagement in lifestyle modification. ${ }^{9}$ Our specific technique consisted of specific goal-setting, prompting self-monitoring, providing feedback on performance, and goal reviewing to increase their engagement. It might lead to meaningful change of behavior and reduction of their geriatric depressive symptoms. ${ }^{26}$ In particular, regular and continuous contacts and attention with symbolic incentives provided by therapists might help decrease their social isolation, a crucial psychodynamic for occurrence of depression in the community dwelling elderly. ${ }^{27,28}$ In addition, results of GDS subscales revealed that this approach could stimulate the lack of motivation in patients with depression in the community.

Our study has some limitations. First, the stability of the effect on geriatric depressive symptoms of LMCM compared to UCM after 12 weeks was unclear. To prove the superiority of sustainability of LMCM over that of UCM, future investigation with a longer examination period is needed. Second, inter-group heterogeneity such as preclinical Alzheimer's disease might have been undetected. Depressive symptoms in subjects might reflect the presence of preclinical Alzheimer's disease. To have a more homogeneous population set in our study, we only included patients over 60 years old with MMSE scores equal or greater than 24 who were cognitively intact. Third, since subjects who participated in this study were enrolled after seeking medical attention in senior citizen centers, results of this study may not reflect the general population. Finally, we did not include factors such as duration of depressive episodes or the rate of relapse/recurrence during treatment. Inclusion of these variables might alter our findings.

In summary, this study demonstrated the effectiveness of lifestyle modification with symbolic incentives in improving geriatric depressive symptoms in older adults in the community. This psychosocial intervention with symbolic incentives might be useful for the elderly who are at risk of having adverse reactions to drugs or with limited response to usual care management in community dwelling older adults. The LMCM is safe and easy to use with a low cost. It can increase social connectedness through continuous attention provided by health workers. It is suitable for older adults with depression in the community. Our results demonstrate that effective psychosocial intervention is important in managing late life depression in older adults in the community. 


\section{Acknowledgments}

This study was supported by a grant (HI15C1032) funded by a R\&D Project of Korea Mental Health Technology.

\section{REFERENCES}

1. Murray CJ, Vos T, Lozano R, Naghavi M, Flaxman AD, Michaud C, et al. Disability-adjusted life years (DALYs) for 291 diseases and injuries in 21 regions, 1990-2010: a systematic analysis for the Global Burden of Disease Study 2010. Lancet 2012;380:2197-2223.

2. Frederick JT, Steinman LE, Prohaska T, Satariano WA, Bruce M, Bryant $\mathrm{L}$, et al. Community-based treatment of late life depression an expert panel-informed literature review. Am J Prev Med 2007;33:222-249.

3. Lackamp J, Schlachet R, Sajatovic M. Assessment and management of major depressive disorder in older adults. Psychiatr Danub 2016; 28(Suppl 1):95-98.

4. Sarris J, O’Neil A, Coulson CE, Schweitzer I, Berk M. Lifestyle medicine for depression. BMC Psychiatry 2014;14:107.

5. Egger GJ, Binns AF, Rossner SR. The emergence of "lifestyle medicine" as a structured approach for management of chronic disease. Med J Aust 2009;190:143-145.

6. Walsh R. Lifestyle and mental health. Am Psychol 2011;66:579-592.

7. van Gool CH, Kempen GI, Penninx BW, Deeg DJ, Beekman AT, van Eijk JT. Relationship between changes in depressive symptoms and unhealthy lifestyles in late middle aged and older persons: results from the Longitudinal Aging Study Amsterdam. Age Ageing 2003;32:81-87.

8. Lee KS, Lee Y, Back JH, Son SJ, Choi SH, Chung YK, et al. Effects of a multidomain lifestyle modification on cognitive function in older adults: an eighteen-month community-based cluster randomized controlled trial. Psychother Psychosom 2014;83:270-278.

9. Alexopoulos GS, Raue PJ, Gunning F, Kiosses DN, Kanellopoulos D, Pollari C, et al. "Engage" therapy: behavioral activation and improvement of late-life major depression. Am J Geriatr Psychiatry 2016;24: 320-326.

10. Steinman LE, Frederick JT, Prohaska T, Satariano WA, Dornberg-Lee $\mathrm{S}$, Fisher R, et al. Recommendations for treating depression in community-based older adults. Am J Prev Med 2007;33:175-181.

11. Lin EH, Von Korff M, Ludman EJ, Rutter C, Bush TM, Simon GE, et al. Enhancing adherence to prevent depression relapse in primary care. Gen Hosp Psychiatry 2003;25:303-310.

12. Ell K. Depression care for the elderly: reducing barriers to evidencebased practice. Home Health Care Serv Q 2006;25:115-148.

13. First MB, Splitzer RL, Gibbon M, Williams JBW. Structured Clinical Interview for DSM-IV-TR Axis I Disorders, Research Version, Patient Edition (SCID-I/P). New York: Biometrics Research, New York State Psychiatric Institute; 2002.

14. Montgomery SA, Asberg M. A new depression scale designed to be sensitive to change. Br J Psychiatry 1979;134:382-389.

15. Lewis MW. Application of contingency management-prize reinforce- ment to community practice with alcohol and drug problems: a critical examination. Behav Soc Issues 2008;17:119-138.

16. Network SIG. Non-Pharmaceutical Management of Depression in Adults: A National Clinical Guideline. Edinburgh: SIGN; 2010.

17. Johnsen M, Samberg L, Calsyn R, Blasinsky M, Landow W, Goldman $\mathrm{H}$. Case management models for persons who are homeless and mentally ill: the ACCESS demonstration project. Access to Community Care and Effective Services and Supports. Community Ment Health J 1999;35:325-346.

18. Simpson A, Miller C, Bowers L. Case management models and the care programme approach: how to make the CPA effective and credible. J Psychiatr Ment Health Nurs 2003;10:472-483.

19. Bae JN, Cho MJ. Development of the Korean version of the Geriatric Depression Scale and its short form among elderly psychiatric patients. J Psychosom Res 2004;57:297-305.

20. Kim JM, Shin IS, Yoon JS, Lee HY. Comparison of diagnostic validaties between MMSE-K and MMSE for screening of dementia. J Korean Neuropsychiatr Assoc 2003;42:124-130.

21. Bernabei R, Landi F, Gambassi G, Sgadari A, Zuccala G, Mor V, et al. Randomised trial of impact of model of integrated care and case management for older people living in the community. BMJ 1998;316:13481351.

22. Gensichen J, von Korff M, Peitz M, Muth C, Beyer M, Guthlin C, et al. Case management for depression by health care assistants in small primary care practices: a cluster randomized trial. Ann Intern Med 2009; 151:369-378.

23. Alexopoulos GS, Raue PJ, McCulloch C, Kanellopoulos D, Seirup JK, Sirey JA, et al. Clinical case management versus case management with problem-solving therapy in low-income, disabled elders with major depression: a randomized clinical trial. Am J Geriatr Psychiatry 2016;24: 50-59.

24. Arean PA, Mackin S, Vargas-Dwyer E, Raue P, Sirey JA, Kanellopolos $\mathrm{D}$, et al. Treating depression in disabled, low-income elderly: a conceptual model and recommendations for care. Int J Geriatr Psychiatry 2010;25:765-769.

25. Arean PA, Raue PJ, McCulloch C, Kanellopoulos D, Seirup JK, Banerjee $S$, et al. Effects of problem-solving therapy and clinical case management on disability in low-income older adults. Am J Geriatr Psychiatry 2015;23:1307-1314

26. Alexopoulos GS, Raue PJ, Kiosses DN, Seirup JK, Banerjee S, Arean PA. Comparing engage with PST in late-life major depression: a preliminary report. Am J Geriatr Psychiatry 2015;23:506-513.

27. Saito T, Kai I, Takizawa A. Effects of a program to prevent social isolation on loneliness, depression, and subjective well-being of older adults: a randomized trial among older migrants in Japan. Arch Gerontol Geriatr 2012;55:539-547.

28. Heikkinen RL, Kauppinen M. Depressive symptoms in late life: a 10year follow-up. Arch Gerontol Geriatr 2004;38:239-250. 